\title{
About one extremal problem for projections of the points on unit circle
}

\author{
ANDREY L. TARGONSKII \\ (Presented by O. A. Dovgoshey)
}

\begin{abstract}
Sharp estimates of product of inner radii for pairwise disjoint domains are obtained. In particular, we solve an extremal problem in the case of arbitrary finite number of free poles on the system points on the rays.
\end{abstract}

2010 MSC. 30C70, 30C75.

Key words and phrases. Inner radius of domain, quadratic differential, piecewise-separating transformation, the Green function, radial systems of points, logarithmic capacity, variational formula.

\section{Introduction}

This paper belongs to the theory of extremal problems on classes of non-overlapping domain, which is a separate direction in geometric theory of functions of a complex variable. The begin of these investigations associated with the paper of M. A. Lavrent'ev [1] in 1934. He found the maximum of some functional with respect to two simply connected domains with two fixed points. We note that this result was needed him for applying to some aerodynamics problems. In 1947, G. M. Goluzin solved a similar problem for three fixed points on the complex plane [2]. Then the topic began to evolve rapidly. In this connection we may recall papers of many authors, including Y. E. Alenitsin, M. A. Lebedev, J. Jenkins, P. M. Tamrazov, P.P. Kufarev and others. Using the idea of P. M. Tamrazov, in 1975 G. P. Bakhtina solved first the problem with so-called "free poles" on the unit circle, see, e.g., [3].

An important step for the development of this topic was papers of V.N. Dubinin. He developed a new method of research that is method

Received 25.05.2018

The author is grateful to Prof. Bakhtin for suggesting problems and useful discussions. 
of piecewise-separating transformation. He also first solved numerous of extremal problems for an arbitrary but multi connected non-overlapping domains (see, e.g., [4-6]). Now this type of extremal problems is used for investigations in holomorphic dynamics.

In the last decade actively used Bakhtin's method of "managing functional". He managed to solve a series of extremal problems for so-called "radial systems of points" (see, e.g., [4, 7-15]). In the present paper we use the mentioned about Bakhtin's method.

Let $\mathbb{N}, \mathbb{R}$ - the sets natural and real numbers conformity, $\mathbb{C}$ - the plain complex numbers, $\overline{\mathbb{C}}=\mathbb{C} \bigcup\{\infty\}$ - the Riemannian sphere, $\mathbb{R}_{+}=(0 ; \infty)$.

For fix number $n \in \mathbb{N}$ system points

$$
A_{n}=\left\{a_{k}\right\}_{k=1}^{n}
$$

the relations are executed:

$$
0=\arg a_{1}<\arg a_{2}<\ldots<\arg a_{n}<2 \pi .
$$

For such systems of points we will consider the following sizes:

$$
\sigma_{k}=\frac{1}{\pi}\left(\arg a_{k+1}-\arg a_{k}\right), k=1,2, \ldots, n, \quad a_{n+1}:=a_{1} .
$$

Let's consider system of angular domains:

$$
M_{k}:=\left\{w: \arg a_{k}<\arg w<\arg a_{k+1}\right\}, \quad k=\overline{1, n}, \quad a_{n+1}:=a_{1}
$$

Let's consider the following "managing functional" for arbitrary $A_{n^{-}}$ system points

$$
\mathrm{T}\left(A_{n}\right)=\prod_{k=1}^{n} \chi\left(\left|\frac{a_{k}}{a_{k+1}}\right|^{\frac{1}{2 \sigma_{k}}}\right)\left|a_{k}\right|,
$$

where $\chi(t)=\frac{1}{2}\left(t+\frac{1}{t}\right)$.

Let $\left\{B_{k}\right\}_{k=1}^{n}$ - arbitrary non-overlapping domains such that

$$
a_{k} \in B_{k}, \quad B_{k} \subset \overline{\mathbb{C}}, \quad k=\overline{1, n} .
$$

Let $D, D \subset \overline{\mathbb{C}}$ - arbitrary open set and $w=a \in D$, then $D(a)$ the define connected component $D$, the contain point $a$. For arbitrary system points $A_{n}=\left\{a_{k} \in \mathbb{C}: k=\overline{1, n}\right\}$, the satisfied condition (1.1), and open set $D, A_{n} \subset D$ the define $D_{k}\left(a_{s}\right)$ connected component set $D\left(a_{s}\right) \cap \overline{M_{k}}$, the contain point $a_{s}, k=\overline{1, n}, s=k, k+1, a_{n+1}:=a_{1}$.

The open set $D, A_{n} \subset D$ satisfied condition meets the condition of unapplied in relation to the system of points $A_{n}$ if a condition is executed

$$
D_{k}\left(a_{k}\right) \bigcap D_{k}\left(a_{k+1}\right)=\varnothing,
$$


$k=\overline{1, n}$, on all corners $\overline{M_{k}}$.

Let

$$
g_{B}(z, a)=h_{B, a}(z)+\log \frac{1}{|z-a|}
$$

generalized Green's function of domains $B$ with respect to a point $a \in B$. If $a=\infty$, then

$$
g_{B}(z, \infty)=h_{B, \infty}(z)+\log \frac{1}{|z|} .
$$

The value of

$$
r(B, a):=\exp \left(h_{B, a}(z)\right)
$$

the define of inner radius domain $B \subset \overline{\mathbb{C}}$ with respect to a point $a \in B$ (see $[4-6,16-18]$ ).

We use the concept of a quadratic differential. Recall that a quadratic differential on a Riemann surface $S$ is a map

$$
\varphi: T S \rightarrow \mathbb{C}
$$

satisfying

$$
\varphi(\lambda v)=\lambda^{2} \varphi(v)
$$

for all $v \in T S$ and all $\lambda \in \mathbb{C}, T S$ - tangent space. If $z \in U \rightarrow \mathbb{C}$, is a chart defined on some open set $U \subset S$ then $\varphi$ is equal on $U$ to

$$
\varphi_{U}(z) d z^{2}
$$

for some function $\varphi_{U}$ defined on $z(U)$.

Suppose that two charts $z: U \rightarrow \mathbb{C}$ and $w: V \rightarrow \mathbb{C}$ on $S$ overlap, and let

$$
h:=w \circ z^{-1}
$$

be the transition function. If $\varphi$ is represented both as $\varphi_{U}(z) d z^{2}$ and $\varphi_{V}(w) d w^{2}$ on $U \cap V$, then we have

$$
\varphi_{V}(h(z))\left(h^{\prime}(z)\right)^{2}=\varphi_{U}(z) .
$$

One way to say this is that quadratic differentials transform under pullbacks by the square of the derivative. As the main results associated with it can be found in [19].

Definition 1.1. A set of points $\left\{\widetilde{a_{k}}=\frac{a_{k}}{\left|a_{k}\right|}: a_{k} \in \mathbb{C}, k=1,2, \ldots, n\right\}$ is called projections of the points on the unit circle. 
Subject of studying of our work are the following problems.

Problem 1. Let $n \in \mathbb{N}, n \geq 2, \alpha \geq 0$. Maximum functional be found

$$
\prod_{k=1}^{n}\left(\left|\widetilde{\mid a_{k+1}}-\widetilde{a_{k}}\right|^{\alpha} \cdot r\left(B_{k}, a_{k}\right)\right)
$$

where $A_{n}=\left\{a_{k}\right\}_{k=1}^{n}$ - arbitrary system points on the rays, the satisfied condition (1.1), $\left\{\widetilde{a_{k}}\right\}_{k=1}^{n}$ - projections of the points on the unit circle, $\left\{B_{k}\right\}_{k=1}^{n}$ - arbitrary set non-overlapping domains, the satisfied condition (1.2), $a_{k} \in B_{k} \subset \overline{\mathbb{C}}$, and all extremal the describe $(k=\overline{1, n})$.

Problem 2. Let $n \in \mathbb{N}, n \geq 2, \alpha \geq 0$. Maximum functional be found

$$
\prod_{k=1}^{n}\left(\left|\widetilde{a_{k+1}}-\widetilde{a_{k}}\right|^{\alpha} \cdot r\left(D, a_{k}\right)\right)
$$

where $A_{n}=\left\{a_{k}\right\}_{k=1}^{n}$ - arbitrary system points on the rays, the satisfied condition (1.1), $\left\{\widetilde{a_{k}}\right\}_{k=1}^{n}$ - projections of the points on the unit circle, $D$ - the open set, the satisfied condition (1.3), $a_{k} \in D \subset \overline{\mathbb{C}}$, and all extremal the describe $(k=\overline{1, n})$.

\section{The case of non-ovellapping domains}

Lemma 2.1. The function

$$
P(\tau)=\ln \sin \frac{\pi \tau}{2}
$$

is convex for $\tau \in(0,2)$.

Proof. Find the second-order derivative

$$
P^{\prime \prime}(\tau)=\frac{\pi}{2} \cdot\left(\operatorname{ctg} \frac{\pi \tau}{2}\right)^{\prime}=-\left(\frac{\pi}{2}\right)^{2} \cdot \frac{1}{\sin ^{2} \frac{\pi \tau}{2}} .
$$

Consequently,

$$
P^{\prime \prime}(\tau)<0 \text {, for } 0<\tau<2 \text {. }
$$

Theorem 2.1. Let $n \in \mathbb{N}, n \geq 2$. Then for all system points $A_{n}=$ $\left\{a_{k}\right\}_{k=1}^{n}$, the satisfied condition (1.1) and arbitrary set non-overlapping domains $\left\{B_{k}\right\}_{k=1}^{n}$, the satisfied condition (1.2), be satisfied inequality

$$
\prod_{k=1}^{n}\left(\left|\widetilde{a_{k+1}}-\widetilde{a_{k}}\right|^{\alpha} \cdot r\left(B_{k}, a_{k}\right)\right) \leq\left(\frac{2^{\alpha+2}}{n} \cdot \sin ^{\alpha} \frac{\pi}{n}\right)^{n} \cdot \mathrm{T}\left(A_{n}\right) .
$$


The equality obtain in this inequality, when points $a_{k}$ and domains $B_{k}$ are, conformity, the poles and the circular domains of the quadratic differential

$$
Q(w) d w^{2}=-\frac{w^{n-2}}{\left(w^{n}-1\right)^{2}} d w^{2} .
$$

Proof. The theorem of the proof leans on a method of the piece-dividing transformation developed by Dubinin (see [4-6]).

Function

$$
\zeta_{k}(w)=-i\left(e^{-i \arg a_{k}} w\right)^{\frac{1}{\sigma_{k}}}, \quad k=1,2, \ldots, n
$$

realizes univalent and conformal transformations of domain $M_{k}$ to the right half-plane $\operatorname{Re} \zeta>0$, for all $k=\overline{1, n}$.

From a formula (2.2) we receive the following asymptotic expressions

$$
\begin{gathered}
\left|\zeta_{k}(w)-\zeta_{k}\left(a_{m}\right)\right| \sim \frac{1}{\sigma_{k}}\left|a_{m}\right|^{\frac{1}{\sigma_{k}}-1}\left|w-a_{m}\right|, \\
w \rightarrow a_{m}, \quad k=1,2, \ldots, n, m=k, k+1 .
\end{gathered}
$$

It's obvious that

$$
\zeta_{k}\left(a_{k}\right)=-i\left|a_{k}\right|^{\frac{1}{\sigma_{k}}}, \zeta_{k}\left(a_{k+1}\right)=i\left|a_{k+1}\right|^{\frac{1}{\sigma_{k}}}, k=1,2, \ldots, n .
$$

Family of functions $\left\{\zeta_{k}(w)\right\}_{k=1}^{n}$, set by equality (2.2), it is possible for by piece-dividing transformation (see [4-6]) domains $\left\{B_{k}: k=\overline{1, n}\right\}$ in relation to the system of corners $\left\{M_{k}\right\}_{k=1}^{n}$. For any domain $\Delta \in \mathbb{C}$ the define $(\Delta)^{*}:=\{w \in \overline{\mathbb{C}}: \bar{w} \in \Delta\}$. Let $G_{k}^{(1)}$ the define connected component $\zeta_{k}\left(B_{k} \cap \bar{M}_{k}\right) \cup\left(\zeta_{k}\left(B_{k} \cap \bar{M}_{k}\right)\right)^{*}$, containing a point $(-i), G_{k-1}^{(2)}-$ the define connected component $\zeta_{k-1}\left(B_{k} \cap \bar{M}_{k-1}\right) \bigcup\left(\zeta_{k-1}\left(B_{k} \cap \bar{M}_{k-1}\right)\right)^{*}$, containing a point $i, k=\overline{1, n}, \bar{M}_{0}:=\bar{M}_{n}, \zeta_{0}:=\zeta_{n}, G_{0}^{(2)}:=G_{n}^{(2)}$. It is clear, that, $G_{k}^{(s)}$ generally speaking, domains are multiconnected domains, $k=\overline{1, n}, s=1,2$. Pair of domains $G_{k-1}^{(2)}$ and $G_{k}^{(1)}$ grows out of piece-dividing transformation domains $B_{k}$ concerning families $\left\{M_{k-1}, M_{k}\right\}$, $\left\{\zeta_{k-1}, \zeta_{k}\right\}$ in point $a_{k}, k=\overline{1, n}$.

From the Theorem 1.9 [16] (see also $[5,6]$ ) and the formulae (2.3), we have the inequalities

$$
\begin{array}{r}
r\left(B_{k}, a_{k}\right) \leq\left[\left|a_{k}\right|^{1-\frac{1}{\sigma_{k}}} \cdot \sigma_{k} \cdot r\left(G_{k}^{(1)}, \zeta_{k}\left(a_{k}\right)\right) \cdot \sigma_{k-1}\right. \\
\left.\times\left|a_{k}\right|^{1-\frac{1}{\sigma_{k-1}}} \cdot r\left(G_{k-1}^{(2)}, \zeta_{k-1}\left(a_{k}\right)\right)\right]^{\frac{1}{2}}, \quad k=1,2, \ldots, n .
\end{array}
$$


From the condition that the points $a_{k}, k=1,2, . ., n$, we get that

$$
\left|\widetilde{a_{k+1}}-\widetilde{a_{k}}\right|=2 \sin \frac{\pi \sigma_{k}}{2}, k=1,2, \ldots, n .
$$

Using formulas (2.5), (2.6) it is received the following ratio:

$$
\begin{aligned}
& \prod_{k=1}^{n}\left(\left|\widetilde{a_{k+1}}-\widetilde{a_{k}}\right|^{\alpha} \cdot r\left(B_{k}, a_{k}\right)\right) \leq 2^{n \alpha} \cdot \prod_{k=1}^{n} \frac{\sigma_{k}\left|a_{k}\right|}{\left|a_{k}\right|^{\frac{1}{2 \sigma_{k}}} \cdot\left|a_{k}\right|^{\frac{1}{2 \sigma_{k-1}}}} \\
\times & \prod_{k=1}^{n} \sin ^{\alpha} \frac{\pi \sigma_{k}}{2} \cdot \prod_{k=1}^{n}\left(r\left(G_{k}^{(1)}, \zeta_{k}\left(a_{k}\right)\right) \cdot r\left(G_{k}^{(2)}, \zeta_{k}\left(a_{k+1}\right)\right)\right)^{\frac{1}{2}} .
\end{aligned}
$$

Inequalities Lavrent'ev using [1] and (2.4), we get:

$$
\begin{aligned}
& r\left(G_{k}^{(1)}, \zeta_{k}\left(a_{k}\right)\right) \cdot r\left(G_{k}^{(2)}, \zeta_{k}\left(a_{k+1}\right)\right) \\
\leq & \left(\left|a_{k}\right|^{\frac{1}{\sigma_{k}}}+\left|a_{k+1}\right|^{\frac{1}{\sigma_{k}}}\right)^{2}, k=1,2, \ldots, n .
\end{aligned}
$$

Taking into account the last inequality, the expression (2.7) can be written as follows:

$$
\begin{gathered}
\prod_{k=1}^{n}\left(\left|\widetilde{a_{k+1}}-\widetilde{a_{k}}\right|^{\alpha} \cdot r\left(B_{k}, a_{k}\right)\right) \\
\leq 2^{n \alpha} \cdot \prod_{k=1}^{n} \sigma_{k} \sin ^{\alpha} \frac{\pi \sigma_{k}}{2} \cdot \prod_{k=1}^{n} \frac{\left|a_{k}\right|^{\frac{1}{\sigma_{k}}}+\left.\left|a_{k+1}\right|^{\frac{1}{\sigma_{k}}}\right|^{\frac{1}{2 \sigma_{k}}} \cdot\left|a_{k}\right|^{\frac{1}{2_{k-1}}}}{\left|a_{k}\right| .}
\end{gathered}
$$

It's obvious that

$$
\prod_{k=1}^{n} \frac{\left|a_{k}\right|^{\frac{1}{\sigma_{k}}}+\left|a_{k+1}\right|^{\frac{1}{\sigma_{k}}}}{\left|a_{k}\right|^{\frac{1}{2_{k}}} \cdot\left|a_{k}\right|^{\frac{1}{2 \sigma_{k-1}}}}\left|a_{k}\right|=2^{n} \cdot \mathrm{T}\left(A_{n}\right) .
$$

Also,

$$
\prod_{k=1}^{n} \sigma_{k} \leq\left(\frac{2}{n}\right)^{n}
$$

The equality obtain in this inequality, if and only if

$$
\sigma_{1}=\sigma_{2}=\ldots=\sigma_{n}=\frac{2}{n} .
$$

Then, we have:

$$
\prod_{k=1}^{n}\left(\left|\widetilde{\mid a_{k+1}}-\widetilde{a_{k}}\right|^{\alpha} \cdot r\left(B_{k}, a_{k}\right)\right) \leq\left(\frac{2^{\alpha+2}}{n}\right)^{n} \cdot \mathrm{T}\left(A_{n}\right) \cdot \prod_{k=1}^{n} \sin ^{\alpha} \frac{\pi \sigma_{k}}{2} .
$$


The equality obtain in this inequality, when points $a_{k}$ and domains $B_{k}$ are, conformity, the poles and the circular domains of the quadratic differential

$$
Q(\zeta) d \zeta^{2}=\frac{d \zeta^{2}}{\left(\zeta^{2}+1\right)^{2}} .
$$

Using the Lemma 2.1 that the function $\alpha \ln \sin \frac{\pi \sigma_{k}}{2}$, is convex for $\sigma_{k} \in(0 ; 2), \alpha \geq 0$. Hence, when $\sigma_{k} \in(0 ; 2)$, then

$$
\frac{\alpha}{n} \cdot \sum_{k=1}^{n} \ln \sin \frac{\pi \sigma_{k}}{2} \leq \alpha \ln \sin \left(\frac{\pi}{2} \cdot \frac{1}{n} \sum_{k=1}^{n} \sigma_{k}\right) .
$$

Given that

$$
\sum_{k=1}^{n} \sigma_{k}=2
$$

we obtain

$$
\prod_{k=1}^{n} \sin ^{\alpha} \frac{\pi \sigma_{k}}{2} \leq \sin ^{n \alpha} \frac{\pi}{n} .
$$

The equality obtain in this inequality, if and only if

$$
\sigma_{1}=\sigma_{2}=\ldots=\sigma_{n}=\frac{2}{n} .
$$

Then from (2.8) using formulas (2.10) it is received the following ratio

$$
\prod_{k=1}^{n}\left(\left|\widetilde{a_{k+1}}-\widetilde{a_{k}}\right|^{\alpha} \cdot r\left(B_{k}, a_{k}\right)\right) \leq\left(\frac{2^{\alpha+2}}{n}\right)^{n} \cdot \mathrm{T}\left(A_{n}\right) \cdot \sin ^{n \alpha} \frac{\pi}{n} .
$$

The equality obtain in this inequality, when points $a_{k}$ and domains $B_{k}$ are, conformity, the poles and the circular domains of the quadratic differential (2.1). It is derived from the square of the quadratic differential (2.9) conversion using

$$
\zeta=-i w^{\frac{n}{2}} .
$$

As a consequence, at $\alpha=0$.

Corollary 2.1. [4] Let $n \in \mathbb{N}, n \geq 2$. Then for all system points $A_{n}=$ $\left\{a_{k}\right\}_{k=1}^{n}$, the satisfied condition (1.1), and arbitrary set non-overlapping domains $\left\{B_{k}\right\}_{k=1}^{n}$, the satisfied condition (1.2), be satisfied inequality

$$
\prod_{k=1}^{n} r\left(B_{k}, a_{k}\right) \leq\left(\frac{4}{n}\right)^{n} \cdot \mathrm{T}\left(A_{n}\right) .
$$


The equality obtain in this inequality, when points $a_{k}$ and domains $B_{k}$ are, conformity, the poles and the circular domains of the quadratic differential (2.1).

As a consequence, at $\alpha=0, \mathrm{~T}\left(A_{n}\right)=1$ we obtain the well known result.

Corollary 2.2. [4-6] Let $n \in \mathbb{N}, n \geq 2$. Then for all system points $A_{n}=\left\{a_{k}\right\}_{k=1}^{n}$, the satisfied condition (1.1) and

$$
\mathrm{T}\left(A_{n}\right)=1,
$$

and arbitrary set non-overlapping domains $\left\{B_{k}\right\}_{k=1}^{n}$, the satisfied condition (1.2), be satisfied inequality

$$
\prod_{k=1}^{n} r\left(B_{k}, a_{k}\right) \leq\left(\frac{4}{n}\right)^{n}
$$

The equality obtain in this inequality, when points $a_{k}$ and domains $B_{k}$ are, conformity, the poles and the circular domains of the quadratic differential (2.1).

\section{The case of open set}

Theorem 3.1. Let $n \in \mathbb{N}, n \geq 2$. Then for all system points $A_{n}=$ $\left\{a_{k}\right\}_{k=1}^{n}$, the satisfied condition (1.1), arbitrary open set $D$, the satisfied condition (1.3), $a_{k} \in D \subset \overline{\mathbb{C}}, k=\overline{1, n}$, be satisfied inequality

$$
\prod_{k=1}^{n}\left(\left|\widetilde{a_{k+1}}-\widetilde{a_{k}}\right|^{\alpha} \cdot r\left(D, a_{k}\right)\right) \leq\left(\frac{2^{\alpha+2}}{n} \cdot \sin ^{\alpha} \frac{\pi}{n}\right)^{n} \cdot \mathrm{T}\left(A_{n}\right) \text {. }
$$

The equality obtain in this inequality, when $D=\bigcup_{k=1}^{n} B_{k}$, where points $a_{k}$ and domains $B_{k}$ are, conformity, the poles and the circular domains of the quadratic differential (2.1).

Proof. At once we will note that from the condition of unapplying follows that cap $\overline{\mathbb{C}} \backslash D>0$ and set $D$ possesses Green's generalized function $g_{D}(z, a)$, where

$$
g_{D}(z, a)=\left\{\begin{array}{l}
g_{D(a)}(z, a), z \in D(a), \\
0, z \in \overline{\mathbb{C}} \backslash \overline{D(a)}, \\
\lim _{\zeta \rightarrow z} g_{D(a)}(\zeta, a), \zeta \in D(a), z \in \partial D(a)
\end{array}\right.
$$


- Green's generalized function open set $D$ concerning a point $a \in D$, and $g_{D(a)}(z, a)$ - Green's function domain $D(a)$ concerning a point $a \in D(a)$.

Further, we will use methods of works $[4,6,7]$. Sets we will consider $E_{0}=\overline{\mathbb{C}} \backslash D ; E\left(a_{k}, t\right)=\left\{w \in \mathbb{C}:\left|w-a_{k}\right| \leqslant t\right\}, \quad k=\overline{1, n}, n \geqslant 2, n \in \mathbb{N}$, $t \in \mathbb{R}_{+}$. The condenser we will enter into consideration for rather small $t>0$

$$
C\left(t, D, A_{n}\right)=\left\{E_{0}, E_{1}\right\},
$$

where

$$
E_{1}=\bigcup_{k=1}^{n} E\left(a_{k}, t\right)
$$

Capacity of the condenser $C\left(t, D, A_{2 n, 2 m-1}\right)$ is called as ( [4], [6])

$$
\operatorname{cap} C\left(t, D, A_{n}\right)=\inf \iint\left[\left(G_{x}^{\prime}\right)^{2}+\left(G_{y}^{\prime}\right)^{2}\right] d x d y
$$

where an infimum undertakes on all continuous and to the lipschicevym in $\overline{\mathbb{C}}$ functions $G=G(z)$, such that $\left.G\right|_{E_{0}}=0,\left.G\right|_{E_{1}}=1$.

Let is named the module of condenser $C$, reverse the capacity of condenser

$$
|C|=[\operatorname{cap} C]^{-1}
$$

From a theorem $1[16]$ get

$$
\left|C\left(t, D, A_{2 n, 2 m-1}\right)\right|=\frac{1}{2 \pi} \cdot \frac{1}{n} \cdot \log \frac{1}{t}+M\left(D, A_{n}\right)+o(1), t \rightarrow 0,
$$

where

$$
M\left(D, A_{n}\right)=\frac{1}{2 \pi} \cdot \frac{1}{n^{2}} \cdot\left[\sum_{k=1}^{n} \log r\left(D, a_{k}\right)+2 \sum_{k \neq q} g_{D}\left(a_{k}, a_{q}\right)\right] .
$$

Function (2.2) and definition $\zeta_{k}\left(a_{k}\right), \zeta_{k}\left(a_{k+1}\right), a_{n+1}, \zeta_{0}, \bar{M}_{0}, \Delta$, $(\Delta)^{*}$, using, by us the theorems entered at proof 1 . Let, too, $\boldsymbol{\Omega}_{k}^{(1)}$ define connected component $\zeta_{k}\left(D \bigcap \bar{M}_{k}\right) \cup\left(\zeta_{k}\left(D \cap \bar{M}_{k}\right)\right)^{*}$, containing a point $\zeta_{k}\left(a_{k}\right)$, a $\Omega_{k-1}^{(2)}-$ connected component $\zeta_{k-1}\left(D \bigcap \bar{M}_{k-1}\right) \bigcup$ $\left(\zeta_{k-1}\left(D \cap \bar{M}_{k-1}\right)\right)^{*}$, containing a point $\zeta_{k-1}\left(a_{k}\right), k=\overline{1, n}, \boldsymbol{\Omega}_{0}^{(2)}:=$ $\boldsymbol{\Omega}_{n}^{(2)}$. It is clear, that $\boldsymbol{\Omega}_{k}^{(s)}$ generally speaking, domains are multiconnected domains, $k=\overline{1, n}, s=1,2$. Pair of domains $\boldsymbol{\Omega}_{k-1}^{(2)}$ and $\boldsymbol{\Omega}_{k}^{(1)}$ grows out of piece-dividing transformation open set $D$ concerning families $\left\{M_{k-1}, M_{k}\right\},\left\{\zeta_{k-1}, \zeta_{k}\right\}$ in point $a_{k}, k=\overline{1, n}$. 
Let's consider condensers

$$
C_{k}\left(t, D, A_{n}\right)=\left(E_{0}^{(k)}, E_{1}^{(k)}\right),
$$

where

$$
E_{s}^{(k)}=\zeta_{k}\left(E_{s} \bigcap \bar{P}_{k}\right) \bigcup\left[\zeta_{k}\left(E_{s} \bigcap \bar{P}_{k}\right)\right]^{*},
$$

$k=\overline{1, n}, s=0,1,\left\{M_{k}\right\}_{k=1}^{n}$ - the system of corners corresponding to system of points $A_{n}$; operation $[A]^{*}$ compares to any the set $A \subset \overline{\mathbb{C}}$ a set, symmetric a set $A$ is relative unit circle $|w|=1$. From this it follows that to the condenser $C\left(t, D, A_{n}\right)$, at dividing transformation is relative $\left\{P_{k}\right\}_{k=1}^{n}$ and $\left\{\zeta_{k}\right\}_{k=1}^{n}$, there corresponds a set of condensers the system of corners corresponding to system of points $A_{n}$; operation $[A]^{*}$ compares to any the set $A \subset \overline{\mathbb{C}}$ a set, symmetric a set $A$ is relative unit circle $|w|=$ 1. From this it follows that to the condenser $C\left(t, D, A_{n}\right)$, at dividing transformation is relative $\left\{M_{k}\right\}_{k=1}^{n}$ and $\left\{\zeta_{k}\right\}_{k=1}^{n}$, there corresponds a set of condensers $\left\{C_{k}\left(t, D, A_{n}\right)\right\}_{k=1}^{n}$, symmetric relatively $\{z:|z|=1\}$. According to works $[4,16]$, we will receive

$$
\operatorname{cap} C\left(t, D, A_{n}\right) \geqslant \frac{1}{2} \sum_{k=1}^{n} \operatorname{cap} C_{k}\left(t, D, A_{n}\right)
$$

From here follows

$$
\left|C\left(t, D, A_{n}\right)\right| \leqslant 2\left(\sum_{k=1}^{n}\left|C_{k}\left(t, D, A_{n}\right)\right|^{-1}\right)^{-1} .
$$

The formula (3.1) gives a module asymptotics $C\left(t, D, A_{n}\right)$ at $t \rightarrow 0$, and $M\left(D, A_{n}\right)$ is the given module of a set $D$ relatively $A_{n}$. Using formulas (2.3) and that fact that a set $D$ meets the condition of unapplied in relation to the system of points $A_{n}$, for condensers we will receive similar asymptotic representations $C_{k}\left(t, D, A_{n}\right), k=\overline{1, n}$

$$
\left|C_{k}\left(t, D, A_{n}\right)\right|=\frac{1}{4 \pi} \log \frac{1}{t}+M_{k}\left(D, A_{n}\right)+o(1), t \rightarrow 0,
$$

where

$$
M_{k}\left(D, A_{n}\right)=\frac{1}{8 \pi} \cdot\left[\log \frac{r\left(\boldsymbol{\Omega}_{k}^{(1)}, \zeta_{k}\left(a_{k}\right)\right)}{\frac{1}{\sigma_{k}}\left|a_{k}\right|^{\frac{1}{\sigma_{k}}-1}}+\log \frac{r\left(\boldsymbol{\Omega}_{k-1}^{(2)}, \zeta_{k-1}\left(a_{k}\right)\right)}{\frac{1}{\sigma_{k-1}}\left|a_{k}\right|^{\frac{1}{\sigma_{k-1}}}-1}\right] .
$$

By means of (3.5), we receive

$$
\left|C_{k}\left(t, D, A_{n}\right)\right|^{-1}=\frac{4 \pi}{\log \frac{1}{t}} \cdot\left(1+\frac{4 \pi}{\log \frac{1}{t}} M_{k}\left(D, A_{n}\right)+o\left(\frac{1}{\log \frac{1}{t}}\right)\right)^{-1}=
$$




$$
=\frac{4 \pi}{\log \frac{1}{t}}-\left(\frac{4 \pi}{\log \frac{1}{t}}\right)^{2} M_{k}\left(D, A_{n}\right)+o\left(\left(\frac{1}{\log \frac{1}{t}}\right)^{2}\right), t \rightarrow 0 .
$$

Further, from (3.6), follows that

$$
\begin{gathered}
\sum_{k=1}^{n}\left|C_{k}\left(t, D, A_{n}\right)\right|^{-1}= \\
\frac{4 \pi n}{\log \frac{1}{t}}-\left(\frac{4 \pi}{\log \frac{1}{t}}\right)^{2} \cdot \sum_{k=1}^{n} M_{k}\left(D, A_{n}\right)+o\left(\left(\frac{1}{\log \frac{1}{t}}\right)^{2}\right), \quad t \rightarrow 0 .
\end{gathered}
$$

In turn, allows (3.7) to receive the following asymptotic representation

$$
\begin{gathered}
\left(\sum_{k=1}^{n}\left|C_{k}\left(t, D, A_{n}\right)\right|^{-1}\right)^{-1} \\
=\frac{\log \frac{1}{t}}{4 \pi n} \cdot\left(1-\frac{4 \pi}{n \log \frac{1}{t}} \cdot \sum_{k=1}^{n} M_{k}\left(D, A_{n}\right)+o\left(\frac{1}{\log \frac{1}{t}}\right)\right)^{-1} \\
=\frac{\log \frac{1}{t}}{4 \pi n}+\frac{1}{n^{2}} \cdot \sum_{k=1}^{n} M_{k}\left(D, A_{n}\right)+o(1), \quad t \rightarrow 0 .
\end{gathered}
$$

Inequalities, (3.3) and (3.4) taking into (3.1) and (3.8) allow to notice that

$$
\frac{1}{2 \pi} \cdot \frac{1}{n} \cdot \log \frac{1}{t}+M\left(D, A_{n}\right)+o(1) \leqslant \frac{\log \frac{1}{t}}{2 \pi n}+\frac{2}{n^{2}} \cdot \sum_{k=1}^{n} M_{k}\left(D, A_{n}\right)+o(1) .
$$

From (3.9) at $t \rightarrow 0$ we receive that

$$
M\left(D, A_{n}\right) \leqslant \frac{2}{n^{2}} \cdot \sum_{k=1}^{n} M_{k}\left(D, A_{n}\right) .
$$

Formulas (3.2), (3.5) and (3.10) lead to the following expression

$$
\begin{gathered}
\frac{1}{2 \pi} \cdot \frac{1}{n^{2}} \cdot\left[\sum_{k=1}^{n} \log r\left(D, a_{k}\right)+2 \sum_{k \neq q} g_{D}\left(a_{k}, a_{q}\right)\right] \\
\leq \frac{1}{4 \pi n^{2}} \cdot\left[\sum_{k=1}^{n} \log \frac{r\left(\boldsymbol{\Omega}_{k}^{(1)}, \zeta_{k}\left(a_{k}\right)\right)}{\frac{1}{\sigma_{k}}\left|a_{k}\right|^{\frac{1}{\sigma_{k}}-1}}+\sum_{k=1}^{n} \log \frac{r\left(\boldsymbol{\Omega}_{k-1}^{(2)}, \zeta_{k-1}\left(a_{k}\right)\right)}{\frac{1}{\sigma_{k-1}}\left|a_{k}\right|^{\frac{1}{\sigma_{k-1}}-1}}\right] .
\end{gathered}
$$


Thus, taking into (2.6), we receive

$$
\begin{aligned}
& \prod_{k=1}^{n}\left(\left|\widetilde{a_{k+1}}-\widetilde{a_{k}}\right|^{\alpha} \cdot r\left(D, a_{k}\right)\right) \leq 2^{n \alpha} \cdot \prod_{k=1}^{n} \frac{\sigma_{k}\left|a_{k}\right|}{\left|a_{k}\right|^{\frac{1}{2 \sigma_{k}}} \cdot\left|a_{k}\right|^{\frac{1}{2 \sigma_{k-1}}}} \\
\times & \prod_{k=1}^{n} \sin ^{\alpha} \frac{\pi \sigma_{k}}{2} \cdot \prod_{k=1}^{n}\left(r\left(\boldsymbol{\Omega}_{k}^{(1)}, \zeta_{k}\left(a_{k}\right)\right) \cdot r\left(\boldsymbol{\Omega}_{k}^{(2)}, \zeta_{k}\left(a_{k+1}\right)\right)\right)^{\frac{1}{2}} .
\end{aligned}
$$

Further, the proof of the theorem comes to an end in the same way, as well as the proof of the theorem 2.1.

\section{References}

[1] M. A. Lavrent'ev, On the theory of conformal mappings // Tr. Fiz.-Mat. Inst. Akad. Nauk SSSR, 5 (1934), 159-245 (in Russian).

[2] G. M. Goluzin, Geometric theory of functions of a complex variable, Nauka, Moscow, 1966 (in Russian).

[3] G. P. Bakhtina Variational methods and quadratic differentials in problems for disjoint domains, $\mathrm{PhD}$ thesis, Kiev, 1975 (in Russian).

[4] A. K. Bakhtin, G. P. Bakhtina, Yu. B. Zelinskii Topological-algebraic structures and geometric methods in complex analysis // Inst. Math. NAS Ukraine, Kiev, 2008 (in Russian).

[5] V. N. Dubinin, Separating transformation of domains and problems of extremal division // Zap. Nauchn. Sem. Leningrad. Otdel. Mat. Inst. Ros. Akad. Nauk. 168 (1988), 48-66 (in Russian).

[6] V. N. Dubinin, Method of symmetrization in the geometric theory of functions of a complex variable // Usp. Mat. Nauk, 49 (1994), No. 1, 3-76.

[7] A. K. Bakhtin, Inequalities for the inner radii of nonoverlapping domains and open sets // Ukr. Math. J. 61 (2009), No. 5, 716-733.

[8] A. K. Bakhtin, A. L. Targonskii Extremal problems and quadratic differential // Nonlin. Oscillations 8 (2005), No. 3, 296-301.

[9] A. L. Targonskii, Extremal problems of partially nonoverlapping domains on a Riemann sphere // Dop. NAN Ukr. 9 (2008), 31-36 (in Russian).

[10] A. Targonskii, Extremal problems on the generalized $(n ; d)$-equiangular system of points // An. St. Univ. Ovidius Constanta, 22 (2014), No. 2, 239-251.

[11] A. Targonskii, Extremal problem (2n;2m-1)-system points on the rays // An. St. Univ. Ovidius Constanta, 24 (2016), No. 2, 283-299.

[12] A. L. Targonskii, Extremal problems for partially non-overlapping domains on equiangular systems of points // Bull. Soc. Sci. Lett. Lodz, 63 (2013), No. 1, $57-63$. 
[13] A. Targonskii, I. Targonskaya, On the One Extremal Problem on the Riemann Sphere // International Journal of Advanced Research in Mathematics, 4 (2016), $1-7$.

[14] A. K. Bakhtin, A. L. Targonskii, Generalized ( $n$, d)-ray systems of points and inequalities for nonoverlapping domains and open sets // Ukr. Math. J., 63 (2011), No. 7, 999-1012.

[15] A. K. Bakhtin, A. L. Targonskii, Some extremal problems in the theory of nonoverlapping domains with free poles on rays // Ukr. Math. J. 58 (2006), No. 12, 1950-1954.

[16] V. N. Dubinin, Asymptotic representation of the modulus of a degenerating condenser and some its applications // Zap. Nauchn. Sem. Peterburg. Otdel. Mat. Inst., 237 (1997), 56-73 (in Russian).

[17] V. N. Dubinin, Capacities of condensers and symmetrization in geometric function theory of complex variables, Dal'nayka, Vladivostok, 2009 (in Russian).

[18] W. K. Hayman, Multivalent functions, Cambridge University, Cambridge, 1958.

[19] J. A. Jenkins, Univalent functions and conformal mapping, Springer, Berlin, 1958.

\section{CONTACT INFORMATION}

Andrey Targonskii Zhitomir State University, Department of Mathematics, Zhitomir, Ukraine

E-Mail: targonsk@zu.edu.ua 